\title{
Effect of Test Coverage and Change Point on Software Reliability Growth Based on Time Variable Fault Detection Probability
}

\author{
Subhashis Chatterjee*, Ankur Shukla \\ Department of Applied Mathematics, Indian School of Mines, Dhanbad, Jharkhand, India. \\ * Corresponding author. Tel.: +91-326-2235441; email: chatterjee_subhashis@rediffmail.com \\ Manuscript submitted July 31, 2015; accepted November 20, 2015. \\ doi: 10.17706/jsw.11.1.110-117
}

\begin{abstract}
In past four decades, many software reliability growth models (SRGMs) have been proposed to enhance the reliability of the software system. During the testing process potential fault sites are sensitized to detect the faults. Fault detection probability increases as learning and maturity of the testing personnel increases. Therefore, in this paper a time variant fault detection probability has been introduced and integrated into s-shaped coverage SRGM. Experimental results shows that the proposed model is better and will be helpful to improve the accuracy of the software reliability estimation.
\end{abstract}

Key words: exponential fault detection probability, non-homogeneous Poisson process (NHPP), software reliability growth model, software reliability, s-shaped testing coverage.

\section{Introduction}

Nowadays, the dependability of the modern society on the software systems is increasing rapidly. Therefore, demand of quality software becomes the most challenging task for software professionals and researchers. Software testing is one of the major software development activity, which helps in improving the quality. The main objective of the software testing is to uncover and removed the inherent and introduced faults with minimum expenditure [1]. Software reliability is the essential part of a quality software, so it is necessary to enhance the reliability of the software. In past four decades, different techniques and methodologies have been developed to improve the quality and reliability of software system, still it is a major concern to estimate the remaining software faults present in a software system accurately. According to Musa [2], 'Software reliability is defined as the failure-free operation of a software under specified environment and specified time'. Many efforts have been made by researchers to estimate and predict the reliability of software through software reliability modeling. In past, various SRGMs have been developed with different approaches and assumptions [3]-[16].

Testing coverage is the one of the important factor which affects the reliability growth of software. It helps in developing more efficient test cases. The main objective of the testing coverage analysis is to find the fault sites which are uncovered and additional test cases required to increase the testing coverage. During the testing process, to detect the faults present at the fault sites, potential fault sites are sensitized [1], [17]. One common assumption of the most of the SRGM that the fault present at the potential fault sites are detected with constant probability [1], [8]. Which is not realistic and reasonable. In reality, fault detection probability increases as the learning and maturity of the tester increases. Therefore, in the 
present study fault detection probability has been considered as exponential function of time. Moreover, different studies have been done considering the various behavior of the testing coverage such as exponential, Weibull, s-shaped etc. [1]. Gokhale et al. [17] concluded that the selection of the coverage function depends on the criteria which fulfills the user's specific requirements. The proposed study has been carried out with the s-shaped coverage factor.

Testing coverage factor and fault detection probability can be affected by different factors such as testing environment, testing strategy, testing team constitution and efficiency, test case effectiveness, resources, etc. Changes in these environmental factors causes change in testing coverage and fault detection probability. These points at which changes are possible is known as 'change point'. The concept of change point first introduced by Zhao [18]. Various SRGMs based on the concept of change point have been proposed by researchers in past few years [19]-[22].

In this paper, a NHPP based SRGM has been developed considering testing coverage. Also, time variant fault detection probability function has been introduced and integrated into the proposed SRGM. To study the effect of the different environment factor on testing coverage factor and fault detection probability, the concept of the change point has been incorporated.

Rest of the article is organized as follows: related work has been presented in Section2. The assumption and formulation of the proposed model has been presented Section 3. Section 4 contains parameter estimation of proposed model and different comparison criteria. Model validation and performance analysis has been carried out in Section 5. Finally, section 6 concludes the work.

\section{Related Work}

Applications of testing coverage has been considered by many researches in software reliability growth modeling [1], [23]. Initially, Gokhale et al. [17] proposed the NHPP based SRGM with testing coverage which is based on the following assumptions:

1) Software faults are distributed uniformly over potential fault sites.

2) When a potential fault site is sensitized, fault present at that site is detected with constant probability.

3) The detected faults are repaired instantaneously without introducing new faults.

They [17] have considered three types of fault coverage functions: exponential, Weibull and s-shaped, and concluded that the selection of testing coverage function will depend on criteria which fulfils the user's requirement. Latter, many SRGMs have been proposed based on the assumptions of Gokhale SRGM [1], [8].

\section{Software Reliability Modeling}

In this section assumptions and formulation of the proposed model with change point and without change point has been presented.

\subsection{Proposed SRGM}

The proposed model is based on the following assumptions:

1) Faults are uniformly distributed over all potential fault sites.

2) Software failures follows an NHPP.

3) When a potential fault site is sensitized at time $t$, any fault present at that site is detected with probability $d(t)$ which increases as learning process increases.

4) The testing coverage function is an s-shaped testing coverage function, i.e., $c(t)=1-(1+b t) \exp (-b t)$ where $b$ is the parameters reflecting the quality of testing.

5) The detected faults are removed immediately without introducing any faults.

From the above assumptions mean value function (MVF) of the proposed model can be obtained by the 
following expression:

$$
\frac{d m(t)}{d t}=a d(t) \frac{d c(t)}{d t}
$$

or

$$
m(t)=a \int_{0}^{t} d(s) c^{\prime}(s) d s
$$

where $a$ is the number of faults which are expected to be detected, $m(0)=0, d(0)=0$ and $c(0)=0$. Since, $d(t)$ increases as learning process increases therefore, it will be an increasing function of time. Let $d(t)=1-\exp (-\beta t)$ where $\beta$ is the positive shape parameter. Solving the equation (2) MVF can be obtained as:

$$
m(t)=a\left[1-\frac{b^{2}}{(b+\beta)^{2}}+\left(\frac{b^{2}}{(b+\beta)^{2}}+\frac{b^{2} t}{b+\beta}-e^{b t}(1+b t)\right) \mathrm{e}^{-(b+\beta) t}\right]
$$

The failure intensity function $\lambda(t)$ can be obtained by differentiating $m(t)$ w.r.t. $t$, i.e.,

$$
\lambda(t)=\frac{d m(t)}{d t}
$$

The conditional reliability of the proposed SRGM can be obtained using the following equation

$$
R(x \mid t)=e^{-[m(t+x)-m(t)]}
$$

\subsection{Proposed Model with Change Point}

Due to change in testing strategy, testing environment, testing effort, defect density, etc., change points can occur in testing coverage factor and fault detection probability. Therefore, coverage factor and the fault detection can be defined with multiple change point as follows:

$$
d(t)=\left\{\begin{array}{l}
1-\exp \left(-\beta_{1} t\right), 0 \leq t \leq \tau_{1} \\
1-\exp \left(-\beta_{2} t\right), \tau_{1} \leq t \leq \tau_{2} \\
: \\
1-\exp \left(-\beta_{n} t\right), t>\tau_{n}
\end{array}\right.
$$

where $\tau_{i}$ is the change point and $\beta_{i}$ is the shape parameter for $i=1,2 \ldots n$.

$$
c(t)=\left\{\begin{array}{l}
1-\left(1+b_{1} t\right) \exp \left(-b_{1} t\right), 0 \leq t \leq \tau_{1} \\
1-\left(1+b_{2} t\right) \exp \left(-b_{2} t\right), \tau_{1} \leq t \leq \tau_{2} \\
: \\
1-\left(1+b_{n} t\right) \exp \left(-b_{n} t\right), t>\tau_{n}
\end{array}\right.
$$

where $\tau_{i}$ is the change point and $b_{i}$ is the parameters reflecting the quality of testing for $i=1,2 \ldots n$. Here, single change point has been considered to reduce the computational complexity.

MVF of the proposed model with single change point $\tau$ can be obtained from the following expression:

$$
m(t)=m(0, \tau]+m(\tau, t]
$$

Using equation (2) and (8) MVF can be written as: 


$$
m(t)=a\left(\int_{0}^{\tau} d_{1}(s) c_{1}^{\prime}(s) d s+\int_{\tau}^{t} d_{2}(s) c_{2}^{\prime}(s) d s\right)
$$

Solving the above expression with $d_{i}(0)=0$ and $c_{i}(0)=0$ for $i=1,2, \mathrm{MVF}$ of the proposed model can be obtained as follows:

$$
m(t)=a\left[\left(\frac{\beta_{2}{ }^{2}}{\left(b_{2}+\beta_{2}\right)^{2}}+\frac{\beta_{2}{ }^{2} t}{b_{2}+\beta_{2}}-e^{b_{2} t}\left(1+\beta_{2} t\right)\right) \mathrm{e}^{-\left(b_{2}+\beta_{2}\right) t}-\left(\frac{\beta_{2}{ }^{2}}{\left(b_{2}+\beta_{2}\right)^{2}}+\frac{\beta_{2}{ }^{2} \tau}{b_{2}+\beta_{2}}-e^{b_{2} \tau}\left(1+\beta_{2} \tau\right)\right) \mathrm{e}^{-\left(b_{2}+\beta_{2}\right) \tau}\right]+m(\tau)
$$

Failure intensity and conditional reliability of the proposed model can be obtained from equation (4) and (5).

\section{Parameter Estimation and Comparison Criteria}

In this section parameter estimation technique of the proposed model and different comparison criteria has been discussed.

\subsection{Parameter Estimation}

The unknown parameters of the models has been estimated with least square technique using SPSS [1]. The position of change point has been obtained using 'changepoint' package in ' $R$ ' software [24].

\subsection{Comparison Criteria}

The following comparison criterion have been used to evaluate the performance of the proposed model.

\subsubsection{Mean square error (MSE)}

It is defined as [7]:

$$
\operatorname{MSE}=\frac{1}{n} \sum_{i=1}^{n}\left(\mathrm{y}_{i}-\hat{\mathrm{y}}_{i}\right)^{2}
$$

where $y_{i}$ and $\hat{y}_{i}$ are the observed and predicted faults respectively, $n$ is the total number of observations.

\subsubsection{Bias}

It is defined as the sum of the deviation of the estimated curve from the actual data, defined as [1]:

$$
\text { Bias }=\frac{1}{n} \sum_{k=1}^{n}\left(m\left(t_{k}\right)-m_{k}\right)
$$

smaller value of bias is better goodness of fit.

\subsubsection{Variance}

It is defined as follows [1]:

$$
\text { Variance }=\sqrt{\frac{1}{n-1} \sum_{i=1}^{n}\left(\mathrm{~m}_{k}-\mathrm{m}\left(\mathrm{t}_{k}\right)-\text { Bias }\right)^{2}}
$$

smaller value of variance is better goodness of fit

\subsubsection{The $100 \mathrm{p} \%$ upper and lower limit for $m(t)$}


It has been given by [25] and defined as follows:

$$
\hat{m}(t)+\eta_{p} \sqrt{\hat{m}(t)} \text { and } \hat{m}(t)-\eta_{p} \sqrt{\hat{m}(t)}
$$

The bounds of $m(t)$ approximatlely as follows:

$$
\hat{m}(t)+\eta_{p} \sqrt{\hat{m}(t)} \geq m(t) \geq \hat{m}(t)-\eta_{p} \sqrt{\hat{m}(t)}
$$

where $\hat{m}(t)$ is the estimate of $m(t)$ and $\eta_{p}$ is the $\frac{(1+p)}{2} \times 100$ percent point of the standard

\section{Model Validation and Performance Analysis}

In this section a numerical example has been shown to evaluate the performance of the proposed model. For this purpose a real software failure data set has been taken which is published in [26] as first data set. This data set contains 481 cumulative number of faults during 111 days test period. The estimated parameters of the proposed model has been given in Table. 2. Change point for this data set is found at position 48.

Table 1. SRGMs with Coverage Factor

\begin{tabular}{|l|l|}
\hline Model & Mean Value Function \\
\hline Proposed model $\left(\mathrm{M}_{1}\right)$ & Equation (3) \\
\hline Proposed model with change point $\left(\mathrm{M}_{2}\right)$ & Equation (10) \\
\hline Exponential coverage model $\left(\mathrm{M}_{3}\right)[17]$ & $m(t)=a(1-\exp (-b t))$ \\
\hline Weibull coverage model $\left(\mathrm{M}_{4}\right)[17]$ & $m(t)=a\left(1-\exp \left(-b t^{k}\right)\right)$ \\
\hline S-shaped coverage model $\left(\mathrm{M}_{5}\right)[17]$ & $m(t)=a(1-(1+b t) \exp (-b t))$ \\
\hline
\end{tabular}

\begin{tabular}{|c|c|c|c|c|c|c|c|c|c|c|}
\hline \multirow[b]{2}{*}{ Model } & \multicolumn{6}{|c|}{ Estimated Parameters } & \multicolumn{4}{|c|}{ Comparison Criteria } \\
\hline & $a$ & $b_{1}$ & $\beta_{1}$ & $b_{2}$ & $\beta_{2}$ & $k$ & MSE & Bias & Variance & $\mathrm{R}^{2}$ \\
\hline $\mathrm{M}_{1}$ & 488.164 & 0.066 & 6.315 & -- & -- & -- & 326.4452 & -1.2998 & 18.2901 & 0.9854 \\
\hline $\mathrm{M}_{2}$ & 481.00 & 0.068 & 1.680 & 0.065 & 5.251 & -- & 320.2596 & -0.1070 & 17.9779 & 0.9856 \\
\hline $\mathrm{M}_{3}$ & 538.070 & 0.026 & -- & -- & -- & -- & 791.9191 & 5.5716 & 27.7091 & 0.9645 \\
\hline $\mathrm{M}_{4}$ & 483.994 & 0.005 & -- & -- & -- & 1.501 & 356.1546 & -6.7148 & 22.2685 & 0.9840 \\
\hline $\mathrm{M}_{5}$ & 489.112 & 0.065 & -- & -- & -- & -- & 332.5846 & -2.5593 & 18.8530 & 0.9851 \\
\hline
\end{tabular}

Table 2. Estimated Parameters and Different Comparison Criteria.

From the Table 2, it is clear that the estimated total number of faults by proposed model without change point is $488.164 \approx 488$ which is very close to the actual number of faults, i.e., 481 . It means 7 faults are still present in the software at the end of the testing. Besides the estimated number of faults by proposed model with change point is 481.00 which is exactly equal to the actual number of faults present in the software. It means no faults are present in the software at end of the testing. As shown in Table 2, testing coverage and fault detection probability increases after change point. This shows the realistic behaviour of testing coverage and fault detection probability. 


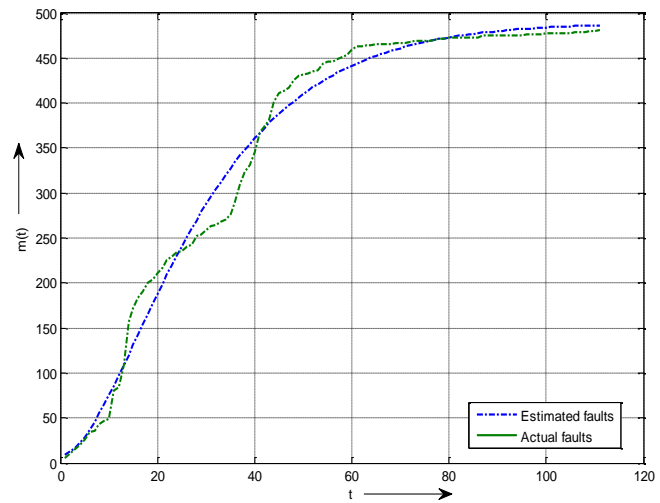

Fig. 1. Estimation of cumulative faults without change point using proposed model.

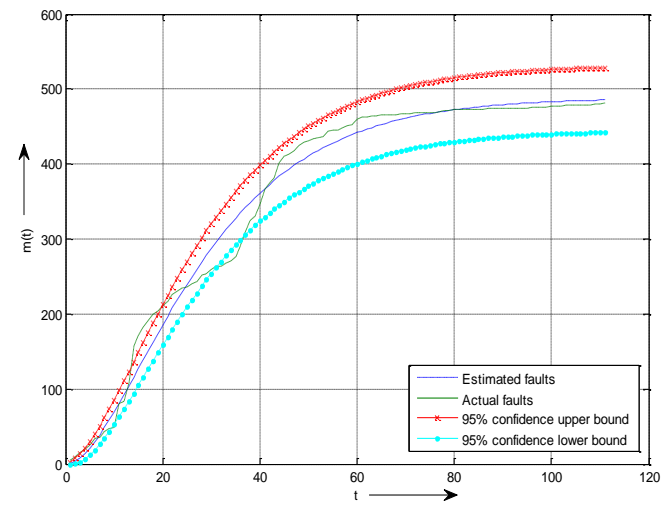

Fig. 2. Estimated cumulative number of faults with $95 \%$ confidence bound.

From Table 2, it is clear that MSE, Bias, Variance and $\mathrm{R}^{2}$ of the proposed models is lowest in comparison to the other models mentioned in Table 1, which shows that the proposed models are better in compare to the other models. While the proposed model with change point is producing lower value of MSE, Bias, Variance and $\mathrm{R}^{2}$ than the proposed model without change point. It means change point plays a significance role to improve the performance of the proposed model. Graphical representation of faults estimated by the proposed model without and with change point has been shown in Fig. 1 and Fig. 3 respectively. 95\% confidence bound of the estimated faults by proposed model without change point and with change point has been shown in Fig. 2 and Fig. 4 respectively. From these figures it is clear that the pattern of the estimated faults are very close to the actual faults. Hence, proposed model is better fit for given data set.

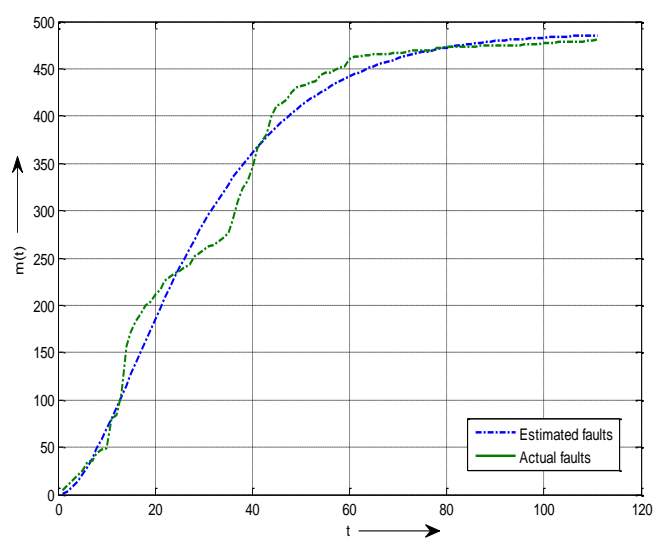

Fig. 3. Estimation of cumulative faults with change point using proposed model.

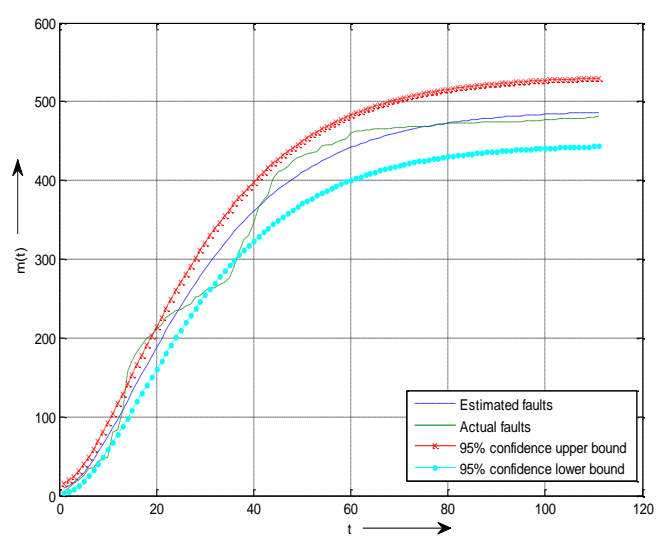

Fig. 4. Estimated cumulative number of faults with $95 \%$ confidence bound.

\section{Conclusion}

In this paper, a SRGM has been proposed with fault coverage and change point introducing the time variant fault detection probability. S-shaped testing coverage function and exponential fault detection probability has been considered in the proposed model. Real software failure data set has been used to validate the proposed model. Experimental results established the fact that the proposed model is better than the other models. Also, the proposed model is more flexible and realistic. Hence, the proposed models can be very helpful for industry and software professionals to improve the quality of software.

\section{Acknowledgements}


Authors acknowledge Indian School of Mines, Dhanbad, India, for providing necessary facilities for this work.

\section{References}

[1] Kapur, P. K., Pham, H., Gupta, A., \& Jha. P. C. (2011). Software Reliability Assessment with OR Application. New York, USA: Springer.

[2] Musa, J. D., Iannino, A., \& Okumoto, K. (1987). Software Reliability, Measurement, Prediction and Application. New York: McGraw-Hill.

[3] Musa, J. D. (1975). A theory of software reliability and its application. IEEE Transactions on Software Engineering, SE-1 (3), 312-327.

[4] Xie, M. (1991). Software Reliability Modeling. Singapore: World Scientific.

[5] Lyu, M. R. (1996). Handbook of Software Reliability Engineering. New York, USA: McGraw-Hill.

[6] Kapur, P. K., Garg, R. B., \& Kumar, S. (1999). Contributions to Hardware and Software Reliability. Singapore: World Scientific.

[7] Pham, H. (2000). Software Reliability. Singapore: Springer-Verlag.

[8] Pham, H. (2006). System Software Reliability. New York: Springer.

[9] Goel, A. L., \& Okumoto, K. (1979). A time-dependent error detection rate model for software reliability and other performance measure. IEEE Transactions on Reliability, R-28(3), 206-211.

[10] Yamada, S., Ohba, M., \& Osaki, S. (1983). S-shaped reliability growth modeling for software error detection. IEEE Transactions on Reliability, R-32(5), 475-484.

[11] Yamada, S., Tokunou, K., \& Osaki, S. (1992). Imperfect debugging models with fault introduction rate for software reliability assessment. International Journal of Systems Science, 23(12), 2253-2264.

[12] Chatterjee, S., Mishra, R. B., \& Alam, S. S. (1997). Joint effect of test effort and learning factor on software reliability and optimal release policy. International Journal of System Science, 28 (4), 391-396.

[13] Yamada, S., Tamura, Y., \& Kimura, M. (1999). A software reliability growth model for a distributed development environment. Transactions of IEICE Japan, J82-A(9), 1446-1453.

[14] Chatterjee, S., Nigam, S., Singh, J. B., \& Upadhyaya, L. N. (2011).Transfer function modeling in software reliability. Computing, 92(1), 33-48.

[15] Chatterjee, S., Nigam, S., Singh, J. B., \& Upadhyaya, L. N. (2011). Application of fuzzy time series in prediction of time between failures and faults in software reliability Assessment, Fuzzy Information and Engineering, 3(3), 293-309.

[16] Chatterjee, S., \& Maji, B. (2015). A new fuzzy rule based algorithm for estimating software faults in early phase of development. Soft Computing.

[17] Gokhale, S. S., Philip, T., Marinos, P. N., \& Trivedi K. S. (1996). Unification of finite failure nonhomogeneous Poisson process models through test coverage. Proceedings of the 7 th International Symposium on Software Reliability Engineering (pp. 299-307).

[18] Zhao, M. (1993). Change-point problems in software and hardware reliability. Communications in Statistics.-Theory and Methods, 22 (3), 757-768.

[19] Chen, M. C., Wu, H. P., \& Shyur, H. J. (2001). Analyzing software reliability growth model with imperfect-debugging and change-point by genetic algorithms. Proceedings of the 29th International Conference on Computers and Industrial Engineering (pp. 520-526).

[20] Shyur, H. J. (2003). A stochastic software reliability model with imperfect debugging and change point. Journal of Systems and Software, 66(2), 135-141.

[21] Inoue S., \& Yamada, S. (2008). Optimal software release policy with change point. Proceedings of the 2008 IEEE International Conference on Industrial Engineering and Engineering Management (pp. 157163). 
[22] Chatterjee, S., Nigam, S., Bahadur, J., \& Upadhyaya, L. N. (2012). Effect of change point and imperfect debugging in software reliability and its optimal release policy. Mathematical and Computer Modelling of Dynamical, 18(5), 1-13.

[23] Chatterjee, S., \& Singh, J. B. (2014). A NHPP based software reliability model and optimal release policy with logistic-exponential test coverage under imperfect debugging. International Journal of Systems Assurance Engineering and Management, 5(3), 399-406.

[24] Killick, R., \& Eckley, I. (2014). Changepoint: An Rpackage for change point analysis. Journal of Statistical Software, 58(3), 1-19.

[25] Yin, L., \& Trivedi, K. S. (1999). Confidence interval estimation of NHPP-based software reliability models. Proceedings of the 10th International Symposium on Software Reliability Engineering (pp. 6-11).

[26] Tohma, Y., Jacoby, R., Murata, Y., \& Yamamoto, M. (1989). Hypergeometric distribution model to estimate the number of residual software faults. Proceedings of the 13th Annual International Computer Software and Applications Conference (pp. 610-617).

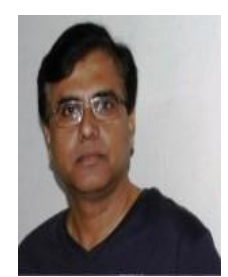

Subhashis Chatterjee was born in 1964, in Bhilai, Chastisgarh, India. He obtained his B.Sc (mathematics) from T.D.B. College, Raniganj, the University of Burdwan, India. He did his M.Sc (mathematics) and Ph.D from IIT Kharagpur, India. He is a member of IEEE Reliability Society. His area of research is software reliability modeling. Presently Dr. Chatterjee is working as an associate professor, in the Dept. of Applied Mathematics, Indian School of Mines (ISM) Dhanbad, India. He has served G.I.E.T, Orissa and SMIT, Sikkim, India, as a faculty. He has total sixteen years of teaching and research experience. He has quite a good number of international and national publications. He has reviewed papers for various national and international journals. His areas of interest are software reliability, web software reliability, O.R., stochastic process, and fuzzy set.

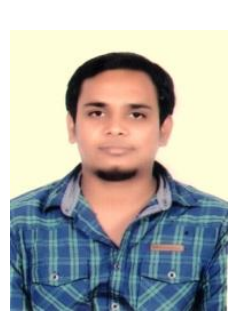

Ankur Shukla was born in 1990, in Ballia district of Uttar Predesh, India. He obtained his B. Sc. (physics and mathematics) from Ewing Christian College, Allahabad, University of Allahabad. He received his M.Sc. degree in mathematics and scientific computing in 2012 from MNNIT, Allahabad, India. He is currently pursuing Ph.D. in the Department of Applied Mathematics from Indian School of Mines Dhanbad, India. His research interests include software reliability modeling, time series, fuzzy time series, genetic algorithm and artificial neural network. 